\title{
CARDIOVASCULAR FUNCTION IN INFANTS BORN SMALL FOR GESTATIONAL AGE AT 2-YEARS
}

Castagno $M^{1}$, Menegon $V^{2}$, Genoni $G^{1}$,Zanetta $S^{1}$, Monzani $A^{1}$, Binotti $M^{3}$, Ferrero $F^{3}$

\author{
1. Division of Pediatrics, Department of Health Sciences, University of Piemonte Orientale, Novara, Italy \\ 2. Vascular Surgery, Maggiore della Carità University Hospital, Novara, Italy \\ 3. Neonatal and Pediatric ICU, Maggiore della Carità University Hospital, Novara, Italy
}

\section{Background \& Aim}

Previous studies have shown an impaired endothelial function in children born small for gestational age (SGA) leading to an increased cardiovascular risk during pediatric and adult age and to long-term morbidity and mortality. However, the age at which cardiovascular damage becomes evident is still not clear and SGA infants do not routinely receive a long-term cardiovascular follow-up.

Aim of this study was to assess clinical and echographic markers of cardiovascular dysfunction in infants born SGA, compared to a control group of subjects born adequate for gestational age (AGA). We evaluated the two groups at 24 months of age, after the physiologic "catch-up growth".

\section{Subjects \& Methods}

This was a monocentric case-control study.

SGA was defined as a birth weight $<10^{\text {th }}$ percentile for gestational age according to Italian neonatal growth charts.

We recruited 20 SGA and 20 sex- and gestational age- matched AGA subjects. All patients were enrolled at 24 months of age.

In the whole population we performed: anthropometric, Doppler two-dimensional echocardiographic assessments, carotid artery intimamedia thickness (CIMT) and endothelium-dependent vasodilation evaluation. End-diastolic pressure (EDP) was calculated from the E/E' ratio using the formula of Nagueh et al. and the pressure-volume curve during diastole using the algorithm for the elastance calculation on one single beat, as proposed by Klotz et al.

\section{Results}

SGA children showed lower stroke volume, left ventricle (LV) dimensions and volume, and greater LV thickness compared with AGA subjects. Diastolic function was impaired in SGA with lower capacitance, and higher E/E' ratio and elastance. Tricuspid annular plane systolic excursion (TAPSE) and TAPSE Z-score were lower in SGA than AGA (Table 1).

Birth weight-SDS was positively associated with TAPSE Z-score and capacitance, and negatively with $E / E^{\prime}$ ratio and elastance.

cIMT and systemic vascular resistance were significantly higher while flow-mediated dilatation (FMD) was lower in SGA compared with AGA (Table 1).

Figure 1 shows the pressure-volume curve during diastole (EDPRV). In SGA infants the curve was shifted to the left compared with AGA: for the same EDP they showed lower end-diastolic volume (EDV), than AGA.

Birth weight-SDS was directly correlated with FMD and inversely with cIMT.

A longer breastfeeding duration was associated with a lower cIMT, even after correction for confounding factors (Figure 2).

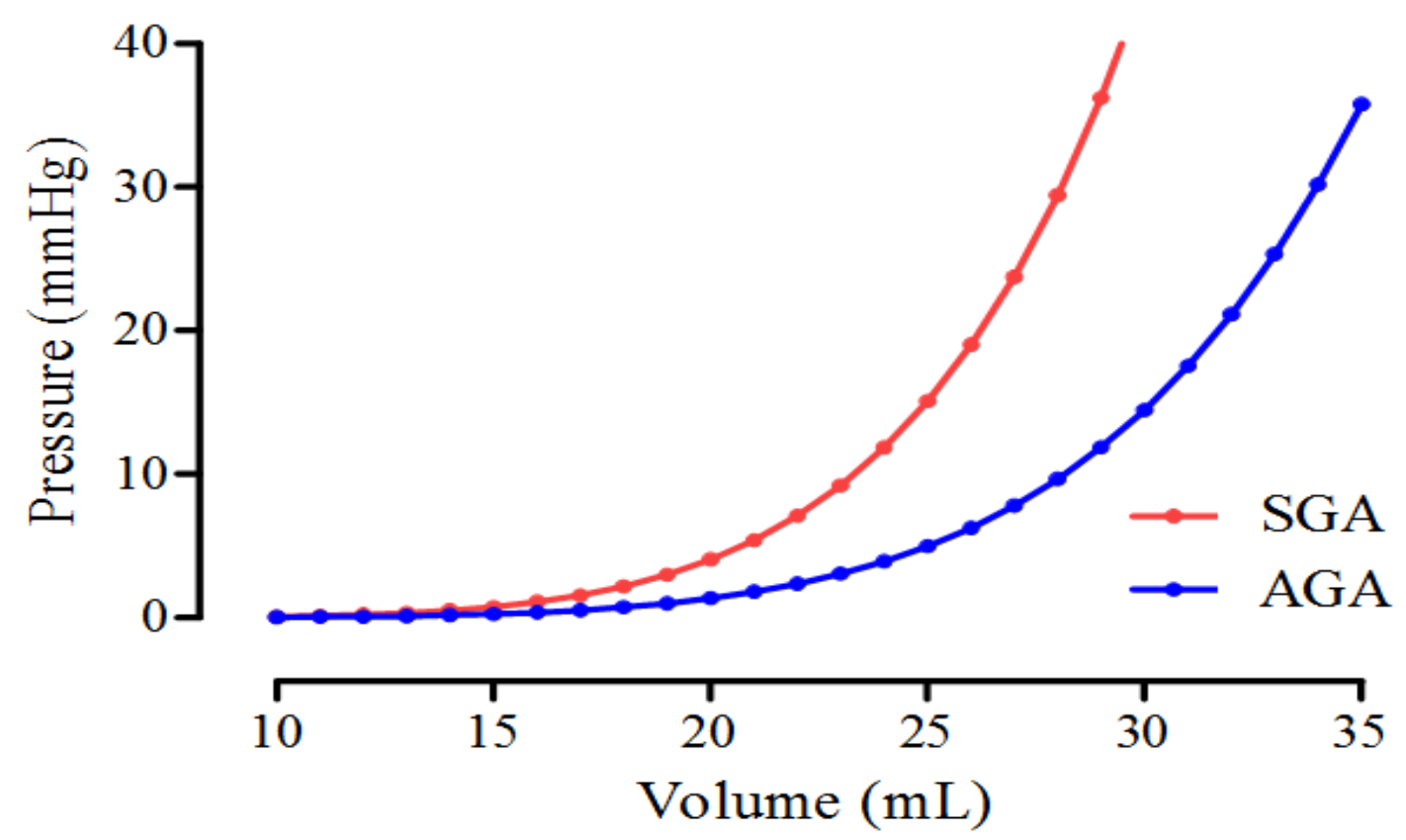

Figure 1. EDPRV curve in 20 SGA vs. 20 AGA subjects

\begin{tabular}{|c|c|c|c|}
\hline & SGA & $\overline{\text { AGA }}$ & p Value \\
\hline Heart rate (bpm) & $118.1 \pm 24.2$ & $115.6 \pm 14.6$ & ns \\
\hline LFEV (\%) Simpson 4C & $59.9 \pm 4.2$ & $62.2 \pm 4.3$ & ns \\
\hline LV stroke volume (mL/Kg) & $1.35 \pm 0.37$ & $1.53 \pm 0.22$ & 0.023 \\
\hline LVO (mL/Kg/min) & $160.1 \pm 57.0$ & $176.1 \pm 27.8$ & ns \\
\hline LVEDD (mm) & $28.5 \pm 2.1$ & $30.4 \pm 2.9$ & 0.013 \\
\hline LVEDD Z-score & $-0.25 \pm 0.86$ & $0.36 \pm 0.69$ & 0.009 \\
\hline LVESD (mm) & $18.6 \pm 1.5$ & $19.8 \pm 1.9$ & 0.025 \\
\hline LVESD Z-score & $0.12 \pm 0.71$ & $0.22 \pm 0.65$ & ns \\
\hline IVSD (mm) & $5.8 \pm 1.0$ & $5.5 \pm 0.8$ & ns \\
\hline IVSD Z-score & $0.99 \pm 0.72$ & $0.62 \pm 0.70$ & 0.049 \\
\hline LVPW (mm) & $4.8 \pm 0.7$ & $4.3 \pm 0.5$ & 0.022 \\
\hline LVPW Z-score & $0.85 \pm 0.73$ & $0.29 \pm 0.58$ & 0.006 \\
\hline LV diastolic volume (mL) & $23.9 \pm 6.4$ & $28.2 \pm 5.3$ & 0.010 \\
\hline LA diastolic volume (mL) & $8.8 \pm 2.2$ & $11.5 \pm 2.1$ & $<0.0001$ \\
\hline E/A ratio & $1.82 \pm 0.56$ & $1.84 \pm 0.51$ & ns \\
\hline E/E' ratio & $8.59 \pm 1.65$ & $7.25 \pm 1.03$ & 0.005 \\
\hline TAPSE (mm) & $16.9 \pm 2.3$ & $19.2 \pm 2.7$ & 0.003 \\
\hline TAPSE Z-score & $0.32 \pm 2.35$ & $1.89 \pm 2.67$ & 0.002 \\
\hline Capacitance $-\mathrm{V}_{30 \mathrm{mmHg}}(\mathrm{mL})$ & $28.13 \pm 7.37$ & $34.03 \pm 6.84$ & 0.006 \\
\hline Elastance - Beta & $5.90 \pm 0.05$ & $5.87 \pm 0.03$ & 0.005 \\
\hline $\mathrm{SBP}(\mathrm{mmHg})$ & $94.7 \pm 8.9$ & $92.4 \pm 7.5$ & ns \\
\hline $\mathrm{DBP}(\mathrm{mmHg})$ & $66.3 \pm 9.1$ & $66.9 \pm 7.6$ & ns \\
\hline cIMT (mm) & $0.48 \pm 0.1$ & $0.39 \pm 0.1$ & 0.003 \\
\hline FMD (\%) & $13.0 \pm 1.5$ & $17.3 \pm 3.5$ & 0.005 \\
\hline SVR (dynes/s/cm²) & $503 \pm 233$ & $411 \pm 85$ & 0.049 \\
\hline
\end{tabular}

Table 1. Cardiovascular parameters at 24 month of age

Values are means \pm SD. LVO, left ventricle output; IVSD, interventricular septal end diastole; LA, left atrium; LV, left ventricle; LVEDD, LV end diastolic diameter; LVEF, LV ejection fraction; LVESD, left ventricle end systolic diameter; TAPSE, tricuspid annular plane systolic excursion, cIMT, carotid intima-media thickness; DBP, diastolic blood pressure; FMD, flow-mediated dilatation; SBP, systolic blood pressure; SVR, systemic vascular resistance.

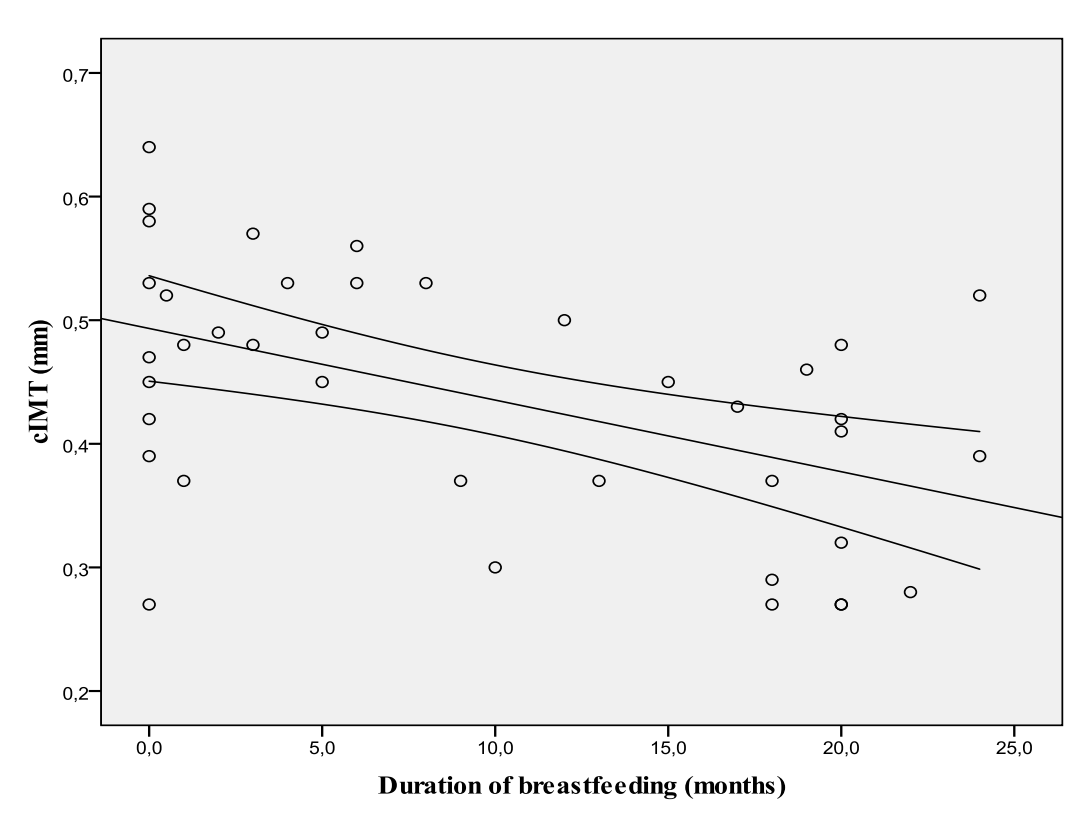

Figure 2. Correlation between breastfeeding duration and cIMT

\section{Conclusions}

Cardiac structure and function and arterial properties are altered in infants born SGA at 2 years of life, confirming the early presence of cardiovascular dysfunction in this group. To precociously identify these alterations is crucial in order to implement preventive and therapeutic measures to stop the progression and improve long-term cardiovascular outcome and mortality. 